\title{
Primeira evidência de Trypanosoma rangeli no sudeste do Brasil, região endêmica para doença de Chagas
}

\author{
First evidence of Trypanosoma rangeli in the South-east \\ of Brazil, an endemic region to Chagas' disease \\ Luis Eduardo Ramirez, Maria Inês Machado, Paula G. Maywald, \\ Aldo Matos, Egler Chiari e Eliane Lages Silva
}

Resumo Informa-se, pela primeira vez, os achados de Trypanosoma rangeli no Triângulo Mineiro, Sudeste do Brasil, área altamente endêmica para doença de Chagas, assim como a infecção natural da espécie Didelphis albiventris.com este mesmo tripanosoma. Estes foram demonstrados por esfregaços sangüíneos, xenodiagnóstico, hemocultura, microhematócrito e PCR. A PCR foi realizada nas fezes e hemolinfa de Triatoma infestans, usando como controle cepas de T. rangeli provenientes da Colômbia.

Palavras-chaves: Trypanosoma rangeli. Didelphis albiventris. Doença de Chagas.

\begin{abstract}
This short communication informs the discovery of Trypanosoma rangeli for the first time at Triângulo Mineiro region, South-east of Brazil, a highly endemic area of Chagas'disease and also the natural infection of Didelphis albiventris with the same trypanosome. Both the findings were demonstrated through blood smears, xenodiagnosis, microhematocrit technics and PCR. The last one was realized in faeces and hemolymph of Triatoma infestans utilizing as controls strains of $\mathrm{T}$. rangeli from Colombia.
\end{abstract}

Key-words: Tripanosoma rangeli. Didelphis albiventris. Chagas' disease.

Departamento de Ciências Biológicas da Faculdade de Medicina do Triângulo Mineiro, Uberaba, MG, Universidade Federal de Uberlândia,Uberlândia, MG, Departamento de Ciências Biológicas da Universidade Federal de Minas Gerais, Belo Horizonte, MG e Companhia Energética de Minas Gerais

Apoio: Companhia Energética de Minas Gerais.

Endereço para correspondência: Dr. Luis Eduardo Ramirez. Disciplina de Parasitologia Departamento de Ciências Biológicas da Faculdade de Medicina do Triângulo Mineiro. Praça Manoel Terra 330, 380015-050 Uberaba, MG .Tel. (034) 3185258.

Recebido para publicação em 21/11/97. 
Trypanosoma rangeli é um parasita hemoflagelado que infecta diversas espécies de hemipteros hematófagos e mamíferos, inclusive o homem. Este protozoário não é considerado patogênico para hospedeiros vertebrados que são infectados pela picada do vetor, principalmente por triatomíneos do gênero Rhodnius 6 . No homem e reservatórios domésticos ou silvestres a parasitemia é baixa e de curta duração, existindo controvérsias relacionadas com sua reprodução. Nos hospedeiros invertebrados naturais invade o hemocele e multiplica-se na hemolinfa, nos hemócitos e posteriormente nas glândulas salivares, podendo lesar o intestino, os túbulos de Malpiglhi, cutícula, traquéia, glândulas salivares e sistema nervoso10 131723 .

A presença simultânea de $T$. rangeli e $T$. cruzi em uma mesma região geográfica possibilita a ocorrência de infecções únicas ou mistas, tanto nos hospedeiros vertebrados como invertebrados 14 dificultando o diagnóstico diferencial das mesmas. Atualmente, recomendase a utilização de diversas metodologias laboratoriais para identificar e caracterizar os isolados primários de $T$. rangeli, bem como para diferencia-lo do T. cruzi, já que podem apresentar reações sorológicas cruzadas 12 . São sugeridos estudos morfológicos dos parasitas, teste de sensibilidade à lise pelo complemento humano e de cobaio, teste de aglutinação com lectina germe de trigo (WGA), imunofluorescência indireta com anticorpos monoclonais, perfil de isoenzimas e reação em cadeia da polimerase (PCR)14911151618192122.

Embora as prevalências humana e animal pelo $T$. rangeli não estejam bem estabelecidas, considera-se ampla a sua distribuição geográfica nas Américas Central e do Sul. No Brasil, foi reportado pela primeira vez por Deane 7 em Didelphis marsupialis no Estado do Pará. Posteriormente, Barrett e Oliveira ${ }^{3}$ o encontraram em ninfas de Rhodnius domesticus na Bahia; Miles e cols 14 reconheceram 46 cepas provenientes de diferentes mamíferos e vetores da bacia Amazônica; D'Alessandro5 encontrou o parasita em macacos Saguinus mistax no Estado de Amazonas e Steindel e cols20 informaram sua presença em roedores Echimys dasythrix no Estado de Santa Catarina. Mais recentemente, foi encontrado em ninfas de $R$. neglectus no Estado de Tocantins 8 .

$\mathrm{Na}$ presente comunicação relatamos pela primeira vez 0 achado de $T$. rangeli na região sudeste do Brasil, denominada Triângulo
Mineiro, área altamente endêmica para doença de Chagas, assim como a infecção natural de marsupiais da espécie Didelphis albiventris com este mesmo tripanosoma.

Dentre a mastofauna resgatada na construção da Usina Hidrelétrica de Miranda (UHM), CEMIG, situada no município de Uberlândia, Minas.Gerais, foram capturados 19 espécimes adultos de $D$. albiventris, dos quais 14 eram fêmeas, três machos e dois mais jovens não tiveram o sexo determinado. Os animais foram transferidos para o Centro de Triagem da UHM, acomodados em gaiolas de madeira grandes e alimentados com ração balanceada. Mais tarde, foram submetidos a exames clínicos, biométricos e, após anestesia, sangrados da veia jugular. Em cada animal foram realizados: exame direto, esfregaço e hemocultura (LIT). Os resultados mostraram $7(36,8 \%)$ hemoculturas positivas e 3 $(15,7 \%)$ esfregaços positivos apresentando tripomastigotas morfologicamente semelhantes a T. cruzi. Seis dos 7 animais positivos para tripanosomatídeos, cinco fêmeas e um macho, foram posteriormente encaminhados à Faculdade de Medicina do Triângulo Mineiro ( FMTM) em Uberaba, onde foram realizados exames complementares, tais como: xenodiagnóstico com ninfas de $3^{\circ}$ e estágio de Triatoma infestans, hemocultura, microhematócrito e PCR. A PCR foi realizada nas fezes e hemolinfa dos triatomíneos com os iniciadores S-35 e S-362 de acordo com as especificações propostas por Vallejo22, utilizando como controle positivo as cepas de $T$. rangeli $\mathrm{CH}$ (Choachi) e P19, ambas isoladas de glândulas salivares de $R$. prolixus na Colômbia e três cepas de $T$. cruzi isoladas de pacientes chagásicos crônicos na região do Triângulo Mineiro.

Os resultados demonstraram parasitemia patente em $100 \%$ dos animais como evidenciado pelo microhematócrito, hemocultura e xenodiagnóstico. O exame da hemolinfa e fezes dos triatomíneos mostraram dois animais com xenodiagnóstico positivo na hemolinfa, três na hemolinfa e fezes e um somente nas fezes. Destes triatomíneos, foram selecionados exemplares de um xenodiagnóstico positivo em fezes e hemolinfa para a realização do PCR, o qual demonstrou amplificação dos fragmentos da região variável de $330 \mathrm{pb}$ do minicírculo do kDNA do T. cruzi, um conjunto de fragmentos de 300 a 450pb e um fragmento específico de 760 pb derivados da região variável do $T$. rangeli, 
evidenciados nos géis de agarose e acrilamida. Estes resultados permitiram concluir que o tripanosoma presente na hemolinfa e fezes dos triatomíneos alimentados em Didelphis albiventris corresponde a $T$. rangeli segundo as especificações de Vallejo22.

\section{REFERÊNCIAS BIBLIOGRÁFICAS}

1. Acosta L, Romanha AJ, Cosenza H, Krettli AU. Trypanosomatid isolates from Honduras: differentia Trypanosoma cruzi and Trypanosoma rangeli. The American Journal Tropical Medicine and Hygiene 44:676-683, 1991.

2. Avila HA, Pereira JB, Thiemann O, De Paiva E, Degrave W, Morel C, Simpson L. Detection of Trypanosoma cruzi in blood specimens of chronic chagasic patients by Polymerase Chain Reaction amplification of kinetoplast minicircle DNA: comparison with serology and xenodiagnosis. Journal of Clinical Microbiology 31:2421-2426, 1993.

3. Barret TV, Oliveira TS. A Trypanosoma indistinguishable from Trypanosoma rangeli in the haemolymph of Rhodnius domesticus from Brazil. The transactions of the Royal Society of Tropical Medicine and Hygiene 71:445-446, 1977.

4. D'Alessandro A. Biology of Trypanosoma (Herpetosoma) rangeli Tejera, 1920. In: Lumsden WHR and Evans DA (eds) Biology of kinetoplastida. Academic Press, London 1:327-403, 1976.

5. D'Alessandro A, Eberhard M, Hincapie D, Halstead S. Trypanosoma cruzi and Trypanosoma rangeli in Saimiri sciureus from Bolivia and Saguinus mistax from Brasil. The American Journal Tropical Medicine and Hygiene 35:285-289, 1986.

6. D'Alessandro A, Hincapie O. Rohodnius neivai: a new experimental vector of Trypanosoma rangeli. The American Journal of Tropical Medicine and Hygiene 35:512-514, 1986.

7. Deane LM. Novo hospedeiro de tripanosomas dos tipos cruzi e rangeli no Estado de Pará. O marsupial Metachirops opossum opossum. Revista Brasileira de Malariologia e Doenças Tropicais 10:531-541, 1958.

8. Diotaiuti I, Silveira AC, Elias M, Steindel M. The possibility of occurrence of Trypanosoma rangeli in the State of Tocantins, Brazil. Memórias Instituto Oswaldo Cruz 87: 451, 1992.

9. Espinosa AG, Taibi A, Billaut-Mulot O, Quaissi A. PCR-based detection of Trypanosoma cruzi useful for specific diagnosis of human Chagas' disease. Journal Clinical Microbiology 34: 485-486, 1996.
10. Garcia ES, Mello CB, Azambuja P, Ribeiro JM. Rhodnius prolixus: salivary antihemostatic components decrease with Trypanosoma rangeli infection. Experimental Parasitology 78:287-293, 1994.

11. Gonçalves AM, Nehme NS, Saraiva N, Segura I, Morel CM. Schizodeme analysis with the restriction endonuclease RSAI differentiates between Trypanosoma rangeli and Trypanosoma cruzi. Memórias Instituto Oswaldo Cruz 86:477-478, 1991.

12. Guhl F. purified Trypanosoma cruzi specific glycoprotein for discriminative serological diagnosis of South American trypanosomiasis (Chagas' disease). Memórias Instituto Oswaldo Cruz 85:531532, 1990.

13. Marinkelle CJ. Trypanosoma rangeli for Rhodnius prolixus stal in nature. Journal Medical Entomology 5:497, 1968.

14. Miles MA, Arias JR, Valente SAS, Naiff RD, Souza AA, Povoa MM, Lima JA, Cedillos RA. Vertebrate hosts and vetors of Trypanosoma rangeli in the Amzon basin of Brazil. The American Journal Tropical Medicine and Hygiene 32:1251-1259, 1983.

15. Miranda Santos IKF, Pereira ME. Lectin discriminate between pathogenic and non pathogenic South American trypanosomes. The American Journal of Tropical Medicine and hygiene 33:839-844, 1984.

16. Ross A, Novoa-Monteiro D. Comparability and reliability of ELISA, immunofluorescence and indirect hemmaglutination assys for Trypanosoma cruzi and Trypanosoma rangeli. Journal Infection Deseases 168:1581-1584, 1993.

17. Schaub GA. The effects of trypanosomatids on insects. In: Dawes b (ed) Advances in parasitology. Academic Press, London 31:255-319, 1992.

18. Schottelius J. Differentiation between Trypanosoma cruzi and Trypanosoma rangeli by their different complement sensitivity. Tropen medizine Parasitenkunden 33:147-150, 1982.

19. Schottelius J, Muller V. Interspecific differentiation of Trypanosoma cruzi, Trypanosoma conorhini and Trypanosoma rangeli by lectins and combvination with complement lysis. Acta Tropica 41:29-38, 1984.

20. Steindel M, Carvalho Pinto JC, Toma HK, Mangia HR, Ribeiro-Rodrigues R, Romanha AJ. Trypanosoma rangeli (Tejera, 1920) isolated from a sylvatic rodent (Echimys dasythrix) in Santa Catarina island, Santa Catarina state: first report of this trypanosome in southern Brazil. Memórias Instituto Oswaldo Cruz 86: 73-79, 1991.

21. Steindel M, Dias Neto E, Ribeiro Rodrigues R, Carvalho Pinto CJ, Grisard EC, Menezes CLP, Murta 
Revista da Sociedade Brasileira de Medicina Tropical 31:99-102, jan-fev, 1998.

SMF, Simpson AGJ, Romanha AJ. Randomly amplified polymorphic DNA (RAPD) and isoenzyme analysis of Trypanosoma rangeli strains. Journal Enkaryotic Microbiology 41: 261-267, 1994.

22. Vallejo GA. Trypanosoma rangeli: Caracterização intra e interespecifica pela análise dos polimorfismos de DNA e kDNA Tese de Doutorado, Universidade
Federal de Minas Gerais, Belo Horizonte,1994.

23. Watkins R. Histology of Rhodnius prolixus infected with Trypanosoma rangeli. Journal Invertebrate Pathology 117: 59-66, 1971. 